\title{
Scalarized system of nonsmooth vector quasi-variational inequalities with applications to Debreu type vector equilibrium problems
}

Mohammed M Alshahrani*

"Correspondence:

mshahrani@kfupm.edu.sa Department of Mathematics and Statistics, King Fahd University of Petroleum and Minerals, KFUPM 1258, Dhahran, 31261, Saudi Arabia

\begin{abstract}
In this work, we utilize a scalarization method to introduce a system of nonsmooth vector quasi-variational inequalities. We also study their relationship to Debreu type vector equilibrium problems. Then we establish some existence results for solutions of these systems by using maximal element theorems for a family of set-valued maps.

Keywords: scalarization; system of nonsmooth vector quasi-variational inequalities; system of Debreu type equilibrium problem for vector-valued functions; system of vector quasi-equilibrium problems; Clarke generalized directional derivative; maximal element theorem; $\Phi$-condensing maps
\end{abstract}

\section{Introduction}

A system of vector quasi-equilibrium problems (SVQEP, for short) is one where we find $\bar{x} \in K$ such that, for each $i \in I$,

$$
\bar{x}_{i} \in A_{i}(\bar{x}) \quad \text { and } \quad \varphi_{i}\left(\bar{x}, y_{i}\right) \notin-\operatorname{int}\left(C_{i}(\bar{x})\right) \quad \text { for all } y_{i} \in A_{i}(\bar{x})
$$

where $I$ is an index set, $K=\prod_{i \in I} K_{i}$ with $K_{i}$ nonempty convex subsets of a Hausdorfflocally convex topological vector space $X_{i}$, and for each $i \in I, A_{i}: K \rightarrow 2^{K_{i}}$ is a set-valued map with nonempty values $\left(2^{K_{i}}\right.$ represents the set of all subsets of $\left.K_{i}\right), \varphi_{i}: K \times K_{i} \rightarrow Y_{i}$, with $Y_{i}$ another Hausdorff locally convex topological vector space, is a bifunction and $C_{i}: K \rightarrow$ $2_{i}^{Y}$ is a set-valued map such that, for each $x \in K, C_{i}(x)$ is a closed, convex, and pointed $\left(C_{i}(x) \cap\left(-C_{i}(x)\right)=\{\mathbf{0}\}\right)$ cone in $Y_{i}$ with a nonempty topological interior, $\operatorname{int}\left(C_{i}(x)\right)$.

This concept of a system of vector quasi-equilibrium problems was first introduced and studied by Ansari et al. [1]. It generalizes the vector equilibrium problem (VEP, for short) which has received a lot of attention by many researchers in recent years [2-7]. This problem is important because it serves as a unified framework for many problems in optimization, such as vector variational inequalities, vector variational-like inequalities, vector complementarity problems, and vector optimization problems. Particularly, vector equilibrium problems are successful in expressing optimality conditions for constrained extremum problems and equilibrium conditions for network flow and economic prob-

\section{黑 Springer}

(c) 2015 Alshahrani; licensee Springer. This is an Open Access article distributed under the terms of the Creative Commons Attribution License (http://creativecommons.org/licenses/by/4.0), which permits unrestricted use, distribution, and reproduction in any medium, provided the original work is properly credited. 
lems; see $[3,8]$ and the references therein. For more on this topic and its applications, we refer the reader to the review paper [9].

This paper is concerned with the study of existence of solutions to an important class of (SVQEP); namely, the Debreu type equilibrium problem for vector-valued functions (DVEP, for short) which seeks to find an $\bar{x} \in K$ such that, for each $i \in I, \bar{x}_{i} \in A_{i}(\bar{x})$, and

$$
f_{i}\left(\bar{x}^{i}, y_{i}\right)-f_{i}(\bar{x}) \notin-\operatorname{int}\left(C_{i}(\bar{x})\right) \quad \text { for all } y_{i} \in A_{i}(\bar{x})
$$

where $f_{i}: K=K^{i} \times K_{i} \rightarrow Y_{i}$ is a vector-valued function and

$$
K^{i}=\prod_{j \in I, j \neq i} K_{j}
$$

Here, we write $x=\left(x^{i}, x_{i}\right)$ for each $x \in K=K^{i} \times K_{i}$ and $\bar{x}$ is said to be a solution of (DVEP).

The following system of problems, called a system of nonsmooth vector quasi-variational inequality problems (SNVQVI, for short), is essential as a tool to study (DVEP). In this system one is interested in finding $\bar{x} \in K$ such that, for each $i \in I$,

$$
\bar{x}_{i} \in A_{i}(\bar{x}) \quad \text { and } \quad h_{i}\left(\bar{x} ; y_{i}-\bar{x}_{i}\right) \notin-\operatorname{int}\left(C_{i}(\bar{x})\right) \quad \text { for all } y_{i} \in A_{i}(\bar{x}) \text {; }
$$

here $h_{i}: K \times K_{i} \rightarrow Y_{i}$ are bifunctions.

In this paper, we introduce a bilinear form that is suitable for the data in (DVEP) and we use it to write (SNVQVI) in a scalarized form. Considering the data of (DVEP), the scalarization method we used is one where we choose a continuous linear functional from a set in the dual space of $Y_{i}$ that is closely related to the ordering cone in $Y_{i}$, then we pair this functional with the range of $f_{i}$. The resulting function, which is nonsmooth, is used to define a bilinear form; namely Clarke's generalized directional derivative. This bilinear form helps finally write a scalarized version of (SNVQVI). Utilizing this method, we are able to establish some existence results for the scalarized version of (SNVQVI) that unify and improve many results in the literature [10-13].

The rest of the paper is organized as follows. Section 2 presents the necessary background needed. In Section 3, we describe the scalarization method and introduce a scalarized system of nonsmooth quasi-variational inequality problems. We also introduce the scalarized Debreu type equilibrium problem for a vector-valued function and investigate its relations to (DVEP) and to the scalarized system of nonsmooth quasi-variational inequality problems. In Section 4, we establish the main results concerning the existence of solutions of the scalarized system of nonsmooth quasi-variational inequality problems.

\section{Definitions and preliminaries}

In this section, we lay out the basic definitions and necessary background required in what follows.

Throughout this section, unless stated otherwise, $X$ and $Y$ are two normed space and $Y^{*}$ is the topological dual of $Y$. Suppose that $K$ is a nonempty subset of $X$ and that $C: K \rightarrow 2^{Y}$ is a set-valued map such that, for each $x \in K, C(x)$ is a closed, convex, and pointed cone in $Y$ with nonempty interior, i.e. $\operatorname{int}(C(x)) \neq \emptyset$, the positive dual of $C$ is a set-valued map $C^{*}: K \rightarrow 2^{Y^{*}}$ defined as

$$
C^{*}(x)=\left\{w \in Y^{*}:\langle w, y\rangle \geq 0 \text { for all } y \in C(x)\right\} \quad \text { for } x \in K .
$$


Although the following result is generally accepted, we include the proof for completeness.

Lemma 2.1 Suppose that $C: K \rightarrow 2^{Y}$ is a set-valued map such that, for each $x \in K, C(x)$ is a closed, convex, and pointed cone in $Y$ with $\operatorname{int}(C(x)) \neq \emptyset$, then

$$
x \in \operatorname{int}(C(\bar{x})) \quad \text { iff } \quad\left\langle w^{*}, x\right\rangle>0 \quad \text { for all } w^{*} \in C^{*}(\bar{x}) \backslash\{\mathbf{0}\} .
$$

Proof Assume that $x \in \operatorname{int}(C(\bar{x}))$ then there exists $\epsilon>0$ such that $x+\epsilon e \in C(\bar{x})$ for all $e \in \mathbb{B}$ where $\mathbb{B}$ is the unit ball. Now assume, to the contrary, that there exists $w_{0}^{*} \in C^{*}(\bar{x}) \backslash\{\mathbf{0}\}$ such that $\left\langle w_{0}^{*}, x\right\rangle=0$, then

$$
\left\langle w^{*}, x+\epsilon e\right\rangle \geq 0 \quad \text { for all } w^{*} \in C^{*}(\bar{x}) \backslash\{\mathbf{0}\} \text { and for all } e \in \mathbb{B},
$$

and, in particular, we have

$$
\left\langle w_{0}^{*}, e\right\rangle \geq 0 \quad \text { for all } e \in \mathbb{B} \text {, }
$$

which implies that $w_{0}^{*}=0$; a contradiction.

Conversely, assume that $\left\langle w^{*}, x\right\rangle>0$ for all $w^{*} \in C^{*}(\bar{x}) \backslash\{\mathbf{0}\}$ and that $x \notin \operatorname{int}(C(\bar{x}))$. Since $\operatorname{int}(C(\bar{x}))$ is a nonempty convex set, then, by the strong separation theorem, there exists a nonzero $w_{0}^{*} \in Y^{*}$ such that

$$
\left\langle w_{0}^{*}, x\right\rangle \leq\left\langle w_{0}^{*}, y\right\rangle \quad \text { for all } y \in \operatorname{int}(C(\bar{x})) .
$$

It is now clear that $\left\langle w_{0}^{*}, y\right\rangle \geq 0$ for all $y \in \operatorname{int}(C(\bar{x}))$. Because, otherwise, if $\left\langle w_{0}^{*}, y_{0}\right\rangle<0$ for some $y_{0} \in \operatorname{int}(C(\bar{x}))$ and since $C(\bar{x})$ is a cone, we can find large enough $\lambda>0$, such that $\left\langle w_{0}^{*}, x\right\rangle>\left\langle w_{0}^{*}, \lambda y_{0}\right\rangle$. Since $C(\bar{x})$ is closed and by the continuity of $w_{0}^{*}$, we have $\left\langle w_{0}^{*}, y\right\rangle \geq 0$ for all $y \in C(\bar{x})$; which shows that $w_{0}^{*} \in C^{*}(\bar{x}) \backslash\{\mathbf{0}\}$. Now if $\left\langle w_{0}^{*}, x\right\rangle>0$ then we can find $z_{0} \in \operatorname{int}(C(\bar{x}))$ and small enough $\mu>0$ such that $\left\langle w_{0}^{*}, x\right\rangle>\left\langle w_{0}^{*}, \mu z_{0}\right\rangle$. Therefore $\left\langle w_{0}^{*}, x\right\rangle \leq 0$. This implies that $\left\langle w_{0}^{*}, x\right\rangle=0$ because $C(\bar{x})$ is pointed. This is a contradiction.

We recall that a set-valued map $\psi: X \rightarrow Y$ is said to be locally Lipschitz at $x \in X$, see [14], if there exist a positive constant $l$ and a neighborhood $U \subset \operatorname{Dom}(\psi):=\{y \in X: \psi(y) \neq \emptyset\}$ of $x$ such that

$$
\psi\left(y_{1}\right) \subset \psi\left(y_{2}\right)+l\left\|y_{1}-y_{2}\right\| \mathbb{B}_{Y} \quad \text { for all } y_{1}, y_{2} \in U,
$$

where $\mathbb{B}_{Y}$ is the unit ball in $Y$. If $\psi$ is locally Lipschitz at every $x \in K$ with $K$ being a nonempty subset of $X$, we say that $\psi$ is locally Lipschitz on $K$. We, also, recall that a realvalued function $\phi: X \rightarrow \mathbb{R}$ from a normed space to the real number line $\mathbb{R}$ is said to be upper semicontinuous on a subset $E$ of $X$ if

$$
\phi(x) \geq \limsup _{y \rightarrow x} \phi(y) \quad \text { for all } x \in E
$$

It is called positively homogeneous on $X$ if $\phi(t x)=t \phi(x)$ for all $x \in X$ and any positive scalar $t$. It is, also, called subadditive on $X$ if for every $x, y \in X, \phi(x+y) \leq \phi(x)+\phi(y)$. A positively homogeneous function is clearly subadditive if and only if it is convex. 
Definition 2.1 (see [15]) Let $\phi: X \rightarrow \mathbb{R}$ be locally Lipschitz at $x \in X$. The Clarke generalized directional derivative of $\phi$ at $x \in X$ in the direction of another vector $d \in X$, denoted by $\phi^{\circ}(x ; d)$, is defined as

$$
\phi^{\circ}(x ; d):=\limsup _{y \rightarrow x, t \rightarrow 0^{+}} \frac{\phi(y+t d)-\phi(y)}{t},
$$

where $y \in X$ and $t$ is a positive number.

The next lemma summarizes the basic properties of the Clarke generalized directional derivative.

Lemma 2.2 (see [15]) Let $\phi: X \rightarrow \mathbb{R}$ be locally Lipschitz at $x \in X$. The following assertions hold true.

(i) The function $\phi^{\circ}(x ; \cdot)$ is finite, positively homogeneous, and subadditive on $X$.

(ii) The function $\phi^{\circ}(\cdot ; \cdot)$ is upper semicontinuous.

Definition 2.2 For an index set $I$, let $X=\prod_{i \in I} X_{i}$ with each $X_{i}$ a normed space and let $\phi_{i}: X \rightarrow \mathbb{R}$ be locally Lipschitz at $x \in X$. The Clarke generalized partial directional derivative of $\phi$ at $x$ in the direction of $d_{i} \in X_{i}$, denoted by $\phi_{i}^{\circ}\left(x ; d_{i}\right)$, is defined to be a Clarke generalized directional derivative of the function $g: X_{i} \rightarrow \mathbb{R}$ defined as

$$
g\left(y_{i}\right)=\phi\left(x^{i}, y_{i}\right) \quad \text { for all } y_{i} \in X_{i},
$$

at $x_{i}$ in the direction of $d_{i}$, where $x_{i}$ is the $i$ th component of $x$ and

$$
x^{i} \in X^{i}=\prod_{j \in I, j \neq i} X_{j}
$$

Remark 2.1 It is clear from the previous definition that the Clarke generalized partial directional derivative inherits all properties of the Clarke generalized directional derivative listed in Lemma 2.2.

Definition 2.3 Let $K$ be a nonempty subset of $X$ and let $\phi: K \rightarrow \mathbb{R}$ be a locally Lipschitz function at $x \in K$. We say that $\phi$ is Clarke-pseudoconvex at $x$ if

$$
\phi^{\circ}(x ; y-x) \geq 0 \quad \text { implies } \quad \phi(y) \geq \phi(x) \quad \text { for all } y \in K .
$$

The function $\phi$ is said to be Clarke-pseudoconvex on $K$ if it is Clarke-pseudoconvex at every point in $K$.

We shall recall the definition of a strictly compactly Lipschitz function. This concept is very essential for scalarizing (SNVQVI).

Definition 2.4 (see [16]) Let $K$ be a nonempty subset of $X$, a function $\psi: K \rightarrow Y$ is said to be strictly compactly Lipschitz at $\bar{x} \in K$ if there exist

1. a set-valued map $\mathfrak{R}: K \rightarrow \operatorname{Comp}(Y)$, where $\operatorname{Comp}(Y)$ is the set of all compact subset of $Y$, 
2. a function $r:(0,1] \times K \times K \rightarrow Y$,

3. a neighborhood $U$ of $\bar{x}$, and

4. a neighborhood $\mathcal{O}$ of 0 in $X$, satisfying the following:

(i) $\lim _{t \rightarrow 0^{+}, x \rightarrow \bar{x}} r(t, x ; v)=0$ for each $v \in \mathcal{O}$ and

$$
\lim _{\substack{t \rightarrow 0^{+} \\ x \rightarrow \bar{x} \\ v \rightarrow 0}} r(t, x ; v)=0
$$

(ii) for all $x \in U, v \in \mathcal{O}$ and $t \in(0,1]$, we have

$$
\frac{\psi(x+t v)-f(x)}{t} \in \mathfrak{R}(v)+r(t, x ; v)
$$

(iii) $\mathfrak{R}(0)=\{0\}$ and the set-valued map $\mathfrak{R}$ is upper semicontinuous at the origin (that is, for each neighborhood of $\mathfrak{R}(0)$ in $Y$ there exists a neighborhood $U$ of 0 in $X$ such that, for each $x \in U, \mathfrak{R}(x) \in W)$.

Moreover, we say that $\psi$ is strictly compactly Lipschitz on $K$ if it is strictly compactly Lipschitz on all $\bar{x} \in K$.

Remark 2.2 In the previous definition, we have the following:

(i) If $Y$ is finite dimensional, then $\psi$ is strictly compactly Lipschitz at $\bar{x}$ if and only if it is locally Lipschitz at $\bar{x}$.

(ii) If $\psi$ is strictly compactly Lipschitz on $K$, then the function $w^{*} \circ \psi: K \rightarrow \mathbb{R}$ defined as

$$
\left(w^{*} \circ \psi\right)(x)=\left\langle w^{*}, \psi(x)\right\rangle \quad \text { for all } x \in K
$$

is locally Lipschitz for all $w^{*} \in Y^{*}$.

Definition 2.5 Let $K$ be a nonempty subset of $X, \psi: K \rightarrow Y$ be a strictly compactly Lipschitz function on $K$, and $w^{*} \in Y^{*}$. We say that $\psi$ is $w^{*}$-Clarke-pseudoconvex if the real-valued function $w^{*} \circ \psi: K \rightarrow \mathbb{R}$ is Clarke-pseudoconvex on $K$, where $\left.\langle\cdot, \cdot\rangle\right\rangle$ is the pairing of $Y^{*}$ and $Y$.

We end this section by giving the mayor tools to establish existence of solutions for (DVEP). Theorem 2.1, due to Deguire et al. [17], is a particular form of a maximal element theorem for a family of set-valued maps.

Definition 2.6 (see [18]) If $E$ is a lattice with a minimal element, denoted by 0 , then a mapping $\Phi: 2^{Z} \rightarrow E$, with $Z$ a Hausdorff topological vector space, is called a measure of noncompactness of $Z$ if the following conditions hold for any $A, B \in 2^{Z}$ :

(i) $\Phi(A)=0$ if and only if $A$ is relatively compact.

(ii) $\Phi(\overline{\operatorname{conv}}(A))=\Phi(A)$, where $\overline{\operatorname{conv}}(A)$ stands for the convex closure of $A$.

(iii) $\Phi(A \cup B)=\max \{\Phi(A), \Phi(B)\}$.

Definition 2.7 (see [18]) Let $L$ and $Z$ be as in Definition 2.6. Suppose $\Phi: 2^{Z} \rightarrow L$ is a measure of noncompactness of $Z$ and $D \subset Z$. An upper semicontinuous (see [14] for a 
definition) set-valued map $T: D \rightarrow 2^{Z}$ is said to be $\Phi$-condensing if the following implication holds:

if $E \subseteq D$ and $\Phi(T(E)) \geq \Phi(E)$, then $E$ is relatively compact.

Remark 2.3 It should be noted that

(i) (iii) in Definition 2.6 implies that if $A \subseteq B$, then $\Phi(A) \leq \Phi(B)$;

(ii) any set-valued map defined on a compact set is $\Phi$-condensing;

(iii) if $Z$ is, also, locally convex, then any compact set-valued map is $\Phi$-condensing for every measure of noncompactness $\Phi$;

(iv) if $T: D \rightarrow 2^{Z}$ is $\Phi$-condensing and $T^{\prime}: D \rightarrow 2^{Z}$ is a set-valued map such that $T^{\prime}(x) \subseteq T(x)$ for all $x \in D$, then $T^{\prime}$ is also $\Phi$-condensing.

Theorem 2.1 Let I be an index set. For each $i \in I$, let $K_{i}$ be a nonempty convex subset of a Hausdorff topological vector space $X_{i}$, and let $G_{i}: K=\prod_{i \in I} K_{i} \rightarrow 2^{K_{i}}$ be a set-valued map. Assume that the following conditions hold:

(i) $G_{i}(x)$ is convex, for all $i \in I$ and all $x \in K$;

(ii) $x_{i} \notin G_{i}(x)$ for all $i \in I$ and all $x \in K$, where $x_{i}$ is the ith component of $x$;

(iii) $G^{-1}\left(y_{i}\right)$ is open for all $i \in I$ and for all $y_{i} \in K_{i}$, and

(iv) there exist a nonempty and compact subset $N$ of $K$ and a nonempty, compact, and convex subset $B_{i}$ of $K_{i}$ for each $i \in I$ such that, for all $x \in K \backslash N$, there exists $i_{0} \in I$ satisfying $G_{i_{0}}(x) \cap B_{i_{0}} \neq \emptyset$.

Then there exists $\bar{x} \in K$ such that $G_{i}(\bar{x})=\emptyset$ for all $i \in I$.

Remark 2.4 If we replace condition (iv) in Theorem 2.1 by the following condition:

(iv) $^{\prime}$ the set-valued map $G: K \rightarrow 2^{K}$, defined as $G(x):=\prod_{i \in I} G_{i}(x)$ for all $x \in K$, is $\Phi$-condensing;

then Theorem 2.1 remains true (see Corollary 4 in [19]) provided that we assume, for each $i \in I$, that $K_{i}$ is nonempty, closed, and convex, and $X_{i}$ is locally convex Hausdorff topological vector space.

\section{Scalarization}

In this section, we utilize the scalarization method introduced by Alshahrani et al. [12] to present scalarized problems of (DVEP) and (SNVQVI) and establish some relations among them. To this end, let $I$ be an index set, $K=\prod_{i \in I} K_{i}$ with $K_{i}$ nonempty convex subsets of a normed space $X_{i}$. Furthermore, we consider, for each $i \in I$, a set-valued map $A_{i}: K \rightarrow 2^{K_{i}}$ with nonempty values, a bifunction $f_{i}: K^{i} \times K_{i} \rightarrow Y_{i}$, with $Y_{i}$ another normed space, and $C_{i}: K \rightarrow 2^{K_{i}}$ is a set-valued map such that $C_{i}(x)$ is a closed, convex, and pointed cone in $Y_{i}$ with nonempty interior for each $x \in K$. We write $x=\left(x^{i}, x_{i}\right) \in K=K^{i} \times K_{i}$, with

$$
x^{i} \in K^{i}=\prod_{j \in I, j \neq i} K_{j}
$$

The scalarized Debreu type equilibrium problem for vector-valued functions (denoted by $w$-DVEP) is to find $\bar{x} \in K$ such that, for each $i \in I, \bar{x}_{i} \in A_{i}(\bar{x})$ and

$$
\begin{aligned}
& \left(w_{i}^{*} \circ f_{i}\right)(\bar{x}) \leq\left(w_{i}^{*} \circ f_{i}\right)\left(\bar{x}^{i}, y_{i}\right) \\
& \quad \text { for all } y_{i} \in A_{i}(\bar{x}) \text { and for some } w_{i}^{*} \in C_{i}^{*}(\bar{x}) \backslash\{\mathbf{0}\} .
\end{aligned}
$$


Proposition 3.1 Every solution of ( $w^{*}$-DVEP) is a solution of (DVEP).

Proof Suppose that $\bar{x}$ is a solution of ( $w^{*}$-DVEP) and assume, to the contrary, that it is not a solution of (DVEP); which means that there exists a $i_{0} \in I$ such that

$$
f_{i_{0}}\left(\bar{x}^{i_{0}}, \hat{y}_{i_{0}}\right)-f_{i_{0}}(\bar{x}) \in-\operatorname{int}\left(C_{i_{0}}(\bar{x})\right) \text { for some } \hat{y}_{i_{0}} \in A_{i_{0}}(\bar{x}) \text {. }
$$

By Lemma 2.1, we have

$$
\left\langle w^{*}, f_{i_{0}}(\bar{x})\right\rangle>\left\langle w^{*}, f_{i_{0}}\left(\bar{x}^{i_{0}}, \hat{y}_{i_{0}}\right)\right\rangle \quad \text { for all } w^{*} \in C_{i_{0}}^{*}(\bar{x}) \backslash\{\mathbf{0}\}
$$

which is a contradiction.

The scalarized system of nonsmooth quasi-variational inequality problems (SSNQVI, for short) is to find $\bar{x} \in K$ such that, for each $i \in I, \bar{x}_{i} \in A_{i}(\bar{x})$, and

$$
\left(w_{i}^{*} \circ f_{i}\right)_{i}^{\circ}\left(\bar{x} ; y_{i}-\bar{x}_{i}\right) \geq 0 \quad \text { for all } y_{i} \in A_{i}(\bar{x}) \text { and for some } w_{i}^{*} \in C_{i}^{*}(\bar{x}) \backslash\{\mathbf{0}\} . \quad \text { (SSNQVI) }
$$

Proposition 3.2 Let $f_{i}: K \rightarrow Y_{i}$ be strictly compactly Lipschitz at $\bar{x} \in K$ and the function $f\left(\bar{x}^{i}, \cdot\right)$ is $w^{*}$-Clarke-pseudoconvex for all $w^{*} \in C_{i}^{*}(\bar{x}) \backslash\{\mathbf{0}\}$. If $\bar{x}$ is a solution to (SSNQVI), then it is a solution to ( $w^{*}$-DVEP).

Proof The proof is immediate from the definition of $w^{*}$-Clarke-pseudoconvexity of $f$ in the second argument.

\section{Main results}

From this point onward, we assume that $I$ is any index set, countable or uncountable, and for each $i \in I, K_{i}$ is a nonempty convex subset of a Hausdorff topological vector space $X_{i}$, $Y_{i}$ is a real locally convex topological vector space, $K=\prod_{i \in I} K_{i}, C_{i}: K \rightarrow 2^{Y_{i}}$ is a set-valued map such that, for all $x \in K, C_{i}(x)$ is a closed, convex, and pointed cone with nonempty interior and $C_{i}^{*}(x)$ is its positive dual, as defined in (1). Furthermore, we assume that, for each $i \in I, A_{i}: K \rightarrow 2^{K_{i}}$ is a set-valued map such that, for all $x \in K, A_{i}(x)$ is nonempty and convex, $A_{i}^{-1}\left(y_{i}\right)$ is open in $K$ for all $y_{i} \in K_{i}$ and the set $\mathfrak{F}_{i}:=\left\{x \in K: x_{i} \in A_{i}(x)\right\}$ is closed in $K$, where $x_{i}$ is the $i$ th component of $x$.

Theorem 4.1 For each $i \in I$, let $f_{i}: K \rightarrow Y_{i}$ be strictly compactly Lipschitz on $K$. Assume that there exist a nonempty and compact subset $N$ of $K$ and a nonempty, compact, and convex subset $B_{i}$ of $K_{i}$ for all $i \in I$ such that, for all $x \in K \backslash N$, there exist $i_{0} \in I$ and $y_{i_{0}} \in B_{i_{0}}$, satisfying $y_{i_{0}} \in A_{i_{0}}(x)$ and

$$
\left(w_{i_{0}}^{*} \circ f_{i_{0}}\right)_{x_{i_{0}}}^{\circ}\left(x ; y_{i_{0}}-x_{i_{0}}\right)<0 \quad \text { for some } w_{i_{0}}^{*} \in C_{i_{0}}^{*}(x) \backslash\{0\} .
$$

Then (SSNQVI) has a solution.

Proof For every $x \in K$ and every $i \in I$, define two set-valued maps $W_{i}: K \rightarrow 2^{K_{i}}$ and $G_{i}$ : $K \rightarrow 2^{K_{i}}$ as follows:

$$
W_{i}(x):=\left\{y_{i} \in K_{i}:\left(w_{i}^{*} \circ f_{i}\right)_{x_{i}}^{\circ}\left(x ; y_{i}-x_{i}\right)<0 \text { for some } w_{i}^{*} \in C_{i}^{*}(x) \backslash\{\mathbf{0}\}\right\}
$$




$$
G_{i}(x):= \begin{cases}A_{i}(x) \cap W_{i}(x), & \text { if } x \in \mathfrak{F}_{i} \\ A_{i}(x), & \text { if } x \in K \backslash \mathfrak{F}_{i} .\end{cases}
$$

Because $\left(w_{i}^{*} \circ f_{i}\right)_{x_{i}}^{\circ}(x ; \cdot)$ is positively homogeneous and subadditive, it follows that $\left(w_{i}^{*} \circ\right.$ $\left.f_{i}\right)_{x_{i}}^{\circ}(x ; \cdot)$ is convex and, therefore, $W_{i}(x)$ is a convex set, which in turn shows that $G_{i}(x)$ is also convex for all $x \in K$ and all $i \in I$. Furthermore, $x_{i} \notin W_{i}(x)$ for all $x \in K$ and all $i \in I$, with $x_{i}$ the $i$ th component of $x$, because the Clarke generalized partial directional derivative vanishes in the direction of the zero vector. This also shows that $x_{i} \notin G_{i}(x)$ for all $x \in K$ and all $i \in I$.

For each $i \in I$ and all $y_{i} \in K_{i}$, the inverse of $G_{i}(x)$ can be written as

$$
G_{i}^{-1}\left(y_{i}\right)=A_{i}^{-1}(x) \cap\left(W_{i}^{-1}\left(y_{i}\right) \cup\left(K \backslash \mathfrak{F}_{i}\right)\right) .
$$

Obviously, $A_{i}^{-1}\left(y_{i}\right)$ and $K \backslash \mathfrak{F}_{i}$ are open. To see that $W_{i}^{-1}\left(y_{i}\right)$ is open, note that the complement of the inverse map of $W_{i}$ in $K$,

$$
\begin{aligned}
{\left[W_{i}^{-1}\left(y_{i}\right)\right]^{c} } & =\left\{x \in K:\left(w_{i}^{*} \circ f_{i}\right)_{x_{i}}^{\circ}\left(x ; y_{i}-x_{i}\right) \geq 0 \text { for some } w_{i}^{*} \in C_{i}^{*}(x) \backslash\{\mathbf{0}\}\right\} \\
& \text { for all } y_{i} \in K_{i}
\end{aligned}
$$

is closed due to the upper semicontinuity of the Clarke generalized partial directional derivative in both arguments. Thus $G_{i}^{-1}\left(y_{i}\right)$ is open for all $i \in I$ and all $y_{i} \in K_{i}$. Therefore by Theorem 2.1, there exists $\bar{x} \in K$ such that $G_{i}(\bar{x})=\emptyset$ for all $i \in I$. Since for each $i \in I$ and all $x \in K, A_{i}(x)$ is assumed to be nonempty, we must have $G_{i}(\bar{x})=A_{i}(\bar{x}) \cap W_{i}(\bar{x})=\emptyset$. In other words, $\bar{x}$ satisfies, for all $i \in I$, the following:

$$
\begin{aligned}
& \bar{x}_{i} \in A_{i}(\bar{x}) \quad \text { and } \\
& \left(w_{i}^{*} \circ f_{i}\right)_{\bar{x}_{i}}^{\circ}\left(\bar{x} ; y_{i}-\bar{x}_{i}\right) \geq 0 \quad \text { for all } y_{i} \in A_{i}(\bar{x}) \text { and for some } w_{i}^{*} \in C_{i}^{*}(\bar{x}) \backslash\{\mathbf{0}\}
\end{aligned}
$$

Thus $\bar{x}$ is a solution of (SSNQVI).

Corollary 4.1 For each $i \in I$, let $f_{i}: K \rightarrow Y_{i}$ be strictly compactly Lipschitz on $K$ and the function $f_{i}\left(\bar{x}^{i}, \cdot\right)$ is $w_{i}^{*}$-Clarke-pseudoconvex for all $w_{i}^{*} \in C_{i}^{*}(x) \backslash\{\mathbf{0}\}$ and all $x \in K$. Assume that there exist a nonempty and compact subset $N$ of $K$ and a nonempty, compact, and convex subset $B_{i}$ of $K_{i}$ for all $i \in I$ such that, for all $x \in K \backslash N$, there exist $i_{0} \in I$ and $y_{i_{0}} \in B_{i_{0}}$, satisfying $y_{i_{0}} \in A_{i_{0}}(x)$ and

$$
\left(w_{i_{0}}^{*} \circ f_{i_{0}}\right)_{x_{i_{0}}}^{\circ}\left(x ; y_{i_{0}}-x_{i_{0}}\right)<0 \quad \text { for some } w_{i_{0}}^{*} \in C_{i_{0}}^{*}(x) \backslash\{0\}
$$

Then (DVEP) has a solution.

Proof The proof follows from Theorem 4.1 and Propositions 3.1 and 3.2.

The following result establishes the existence of solutions to (SSNQVI) in the presence of $\Phi$-condensing maps. 
Theorem 4.2 For each $i \in I$, let $f_{i}: K \rightarrow Y_{i}$ be strictly compactly Lipschitz on $K$ and let the set-valued map $A: K \rightarrow 2^{K}$, defined as

$$
A(x)=\prod_{i \in I} A_{i}(x) \quad \text { for } x \in K,
$$

be $\Phi$-condensing. Then (SSNQVI) has a solution.

Proof In light of Remark 2.4, we only need to show that the set-valued map $G: K \rightarrow 2^{K}$, defined by

$$
G(x)=\prod_{i \in I} G_{i}(x) \quad \text { for } x \in K,
$$

where the $G_{i}$ are as defined in the proof of Theorem 4.1, is $\Phi$-condensing. From the definition of $G_{i}$, we have $G_{i}(x) \subseteq A_{i}(x)$ for all $i \in I$ and all $x \in K$ and therefore $G(x) \subseteq A(x)$ for all $x \in K$. Because $A$ is $\Phi$-condensing, it follows from (iv) and Remark 2.3 that $G$ is $\Phi$-condensing as well.

Remark 4.1 Theorem 4.1 and Theorem 4.2 improve and generalize many existence results in the literature; see for example Theorem 9 in [10], Theorem 4.1 in [11], Theorem 2 in [12] and Theorem 1 in [13].

\section{Competing interests}

The author declares to have no competing interests.

\section{Acknowledgements}

The author is thankful to King Fahd University of Petroleum and Minerals (KFUPM), Dhahran Saudi Arabia for providing excellent research facilities to carry out this research. This work is supported by KFUPM through internally university funded research grant number SP121035 (IN121035).

Received: 16 February 2015 Accepted: 1 April 2015 Published online: 11 April 2015

\section{References}

1. Ansari, QH, Chan, WK, Yang, XQ: The system of vector quasi-equilibrium problems with applications. J. Glob. Optim. 29(1), 45-57 (2004)

2. Ansari, QH: Existence of solutions of systems of generalized implicit vector quasi-equilibrium problems. J. Math. Anal. Appl. 341(2), 1271-1283 (2008)

3. Giannessi, F (ed.): Vector Variational Inequalities and Vector Equilibria: Mathematical Theories. Nonconvex Optimization and Its Applications, vol. 38. Springer, Berlin (2000)

4. Hadjisavvas, N, Schaible, S: From scalar to vector equilibrium problems in the quasimonotone case. J. Optim. Theory Appl. 96(2), 297-309 (1998)

5. Hou, SH, Yu, H, Chen, GY: On vector quasi-equilibrium problems with set-valued maps. J. Optim. Theory Appl. 119(3), 485-498 (2003)

6. Kimura, K, Liou, Y-C, Shyu, DS, Yao, J-C: Simultaneous system of vector equilibrium problems. J. Ind. Manag. Optim. 5(1), 161-174 (2009)

7. Wang, SH, Fu, JY: Stampacchia generalized vector quasi-equilibrium problem with set-valued mapping. J. Glob. Optim. 44(1), 99-110 (2009)

8. Mastroeni, G: On the image space analysis for vector quasi-equilibrium problems with a variable ordering relation. J. Glob. Optim. 53(2), 203-214 (2012)

9. Bigi, G, Castellani, M, Pappalardo, M, Passacantando, M: Existence and solution methods for equilibria. Eur. J. Oper. Res. 227(1), 1-11 (2013)

10. Santos, LB, Rojas-Medar, M, Ruiz-Garzón, G, Rufián-Lizana, A: Existence of weakly efficient solutions in nonsmooth vector optimization. Appl. Math. Comput. 200(2), 547-556 (2008)

11. Al-Homidan, S, Alshahrani, M, Ansari, QH: System of nonsmooth variational inequalities with applications. Optimization 64, 1211-1218 (2015)

12. Alshahrani, M, Ansari, QH, Al-Homidan, S: Nonsmooth variational-like inequalities and nonsmooth vector optimization. Optim. Lett. 8(2), 739-751 (2014)

13. Alshahrani, M: Nonsmooth quasi-variational-like inequalities with applications to nonsmooth vector quasi-optimization. Filomat (2015, accepted) 
14. Aubin, J-P, Frankowska, H: Set-Valued Analysis. Modern Birkhäuser Classics. Birkhäuser Boston, Boston (1990)

15. Clarke, FH: Optimization and Nonsmooth Analysis. Canadian Mathematical Society Series of Monographs and Advanced Texts. Wiley, New York (1983)

16. Thibault, L: On generalized differentials and subdifferentials of Lipschitz vector-valued functions. Nonlinear Anal., Theory Methods Appl. 6(10), 1037-1053 (1982)

17. Deguire, $P$, Tan, KK, Yuan, GX-Z: The study of maximal elements, fixed points for $L_{s}$-majorized mappings and their applications to minimax and variational inequalities in product topological spaces. Nonlinear Anal., Theory Methods Appl. 37(7), 933-951 (1999)

18. Fitzpatrick, PM, Petryshyn, WV: Fixed point theorems for multivalued noncompact acyclic mappings. Pac. J. Math. 54(2), 17-23 (1974)

19. Chebbi, S, Florenzano, M: Maximal elements and equilibria for condensing correspondences. Nonlinear Anal., Theory Methods Appl. 38(8), 995-1002 (1999)

Submit your manuscript to a SpringerOpen ${ }^{\circ}$ journal and benefit from:

- Convenient online submission

Rigorous peer review

- Immediate publication on acceptance

- Open access: articles freely available online

- High visibility within the field

- Retaining the copyright to your article 